\title{
Performance Analysis of T-Wave-Offset Detection Algorithms on Patients With Cardiac Diseases
}

\author{
Deborah Nairn ${ }^{1,2}$, Pietro Bonizzi ${ }^{1}$, Joël Karel ${ }^{1}$, Alfonso Aranda ${ }^{2}$ \\ ${ }^{1}$ Department of Data Science and Knowledge Engineering, Maastricht University, The Netherlands \\ ${ }^{2}$ Medtronic Bakken Research Center, Maastricht, The Netherlands
}

\begin{abstract}
Cardiovascular diseases are the number one cause of death in the world. The application of automatic processing algorithms can provide important information about these heart diseases. However, the design of these algorithms can be challenging due to the morphological variations in ECG signals, specifically in the T-wave-offset. This study proposes a comparison of several T-offset detection algorithms on healthy subjects and patients suffering from cardiac diseases. Seven state of the art algorithms were selected for implementation and were evaluated using the same dataset and benchmark to provide a fair comparison. Although no algorithm performs with $100 \%$ accuracy for all patients, most can perform well with regards to the healthy patients, with two algorithms having a high performance, above $70 \%$ accuracy, on all patients.
\end{abstract}

\section{Introduction}

Cardiovascular diseases (CVD) is the leading cause of death in the world, accounting for 17.7 million deaths in 2015 [1]. Therefore, cardiac research is important to improve cardiovascular diagnosis, specifically automatic processing of the ECG. By detection and delineation of the waveforms of the ECG: P-wave, QRS-complex and Twaves, their duration and amplitude can be found and used to discover heart abnormalities [2]. Due to the extent of conditions which cause irregular T-waves, the research for T-wave detection algorithms is gaining significance within ECG diagnostics [3]. The T-offset is for many diseases an important fiducial point to detect, as well as the most difficult. For instance, coronary heart disease can cause a prolonged QT interval (Q-onset to T-offset) usually indicating increased vulnerability to malignant ventricle arrhythmias and sudden death [4].

With a lot of variation occurring in ECG signals, the ability to create a generic automatic algorithm for detecting the fiducial points of each wave is a difficult task. Many algorithms have been designed over the years which can de- tect the T-offset [7-12]. However, they are typically validated on patients with healthy heart signals where the morphologies of the waves are standard [5]. The aim of this study is therefore to identify how these algorithms perform on patients who are suffering from different cardiac diseases and how they perform in comparison to each other.

\section{Methods}

Seven state of the art T-offset detection algorithms were selected for implementation based on the type of method being used and the clarity of techniques presented in the paper. Each algorithm is explained in a short summary stating the methods used and presented under the lead authors name. Due to some ambiguity about the technical details of the methods used, assumptions have been made which may have caused slight changes in the algorithms.

\subsection{Laguna}

Based on the previous paper of Laguna 1990 [6] this paper takes the same concept of filtering and thresholding with some alterations making it applicable for multilead waveform determination. The T-offset is determined as the point which crosses a threshold to the right of the T-peak. The threshold is determined by the equation: Threshold $=\operatorname{Der}(p k) / k$, where $k$ is a set value which was experimentally adjusted by the authors and chosen as it gave the best performance. $\operatorname{Der}(p k)$ is the value of the derivative signal at time instance of the adjacent peak $p k$ to the right of the T-peak. The code was supplied via PhysioNet [7].

\subsection{Daskalov}

This algorithm considers a different approach for finding the fiducial points of the T-wave, by using a concept of "wings" which is a method of taking two adjacent segments of equal sizes from a point and shifting through an interval. To find the T-offset, two points are calculated. First $T_{i s}$, which is located in a window $10 \mathrm{~ms}$ from the 
peak, is found as the point where $\left(S_{i-5}-S_{i}\right)\left(S_{i}-S_{i+5}\right)<$ $3 * 10^{-6} . S$ is the signal at the $i^{t h}$ position. The amplitude of the wave is then calculated by taking absolute value of the height of the T-peak minus the height of $T_{i s}$. Using this information the equation $T_{s}=0.2 * T_{a m p}$ can be applied and a window from $T_{s}$ to $T_{i s}$ is taken. The wings function is then applied with $10 \mathrm{~ms}$ segments, and the minimum of the function is taken as the T-offset [8].

\subsection{Last}

Three approaches were proposed within this paper to allow for self-comparison. Since the focus is on detecting the T-offset fiducial point, only two out of the three approaches were implemented: the Non-Syntactic Approach and Multi-Component Cross Correlation Approach (MCC) [9].

2.3.0.a Non-Syntactic Approach For this approach, the T-offset is found by taking a window from the T-peak and calculating the gradient of the signal. The T-offset is then determined to be the point when the signal goes below a threshold of $25 \%$ of the maximum gradient of the T-wave, for a duration of $25 \mathrm{~ms}$.

2.3.0.b MCC Approach The second approach of this paper then uses templates of the T-wave and calculates the cross correlation compared with the signal. The point with the best correlation between the beat in the signal and the template, provides the location of the T-wave for every beat. It is assumed that the end location of the template is the T-offset when applied to the signal.

\subsection{Vazquez-Seisdedos}

This method for T-offset detection is based on calculating the area of a trapezium with three fixed vertexes, $\left(x_{m}, y_{m}\right),\left(x_{r}, y_{r}\right),\left(x_{r}, y_{m}\right)$ and with one mobile one $\left(x_{i}, y_{i}\right)$. On a beat for beat process: the point $\left(x_{m}, y_{m}\right)$ is found taking a window from the T-peak to $200 \mathrm{~ms}$ after, and is defined as the point which has the highest absolute derivative. $\left(x_{r}, y_{r}\right)$ is then found in a window of $200 \mathrm{~ms}$ to $400 \mathrm{~ms}$ from the peak and is the point where the derived signal is closest to zero. $\left(x_{i}, y_{i}\right)$ is then moved between $\left(x_{m}, y_{m}\right)$ and $\left(x_{r}, y_{r}\right)$. The area is calculated for each sample point via the equation: $A=0.5\left(y_{m}-y_{i}\right)\left(2 x_{r}-\right.$ $\left.x_{i}-x_{m}\right)$. The T-offset is then identified as the point with the maximum area [10].

\subsection{Madeiro}

The algorithm of Madeiro [11] uses mathematical models of a skewed Gaussian function. A template is created based on a Gaussian function which is modified by a mathematical procedure to insert asymmetry to be able to model the T-wave. Cross-correlation is then used to find the Twaves and by locating the maximum amplitude, the T-peak is found for each beat. Using the T-peak locations, the Toffset is then found by using a slightly adapted version of the Trapezium Area method proposed by Vazquez.

\subsection{Cabasson}

This paper presents a Time Delay Estimation technique of each T-wave with respect to a template wave, the method follows an improved version of Woody's method [12]. The paper simply describes the theoretical formulation of the improved Woody's method for TDE to show that the initial Woody's method is not optimal. The code that was obtained is known to be a modification of the original one, with instead of the user providing manually a window of analysis for the T-wave, the code now uses a Gaussian fitting of the averaged T-wave obtained in the signal.

\section{Databases}

The algorithms selected were evaluated on three data sets. First, using an ECG simulator, constructed by the principle of Fourier series, which was developed and obtained via MathWorks [13]. This allows ECG recordings to be produced without the corruption of noise or disturbance that often occur from machines. For the analysis of the algorithms, the simulator was used to create 15 different ECG recordings: with different heart rates, T-wave amplitudes and white noise. Therefore, each algorithm can be assessed on which characteristics cause a reduction in performance.

The second database used was PhysioNet's PTB Diagnostic ECG Database, which contains 268 records from a range of different aged patients both male and female. The recordings contain various diagnostics, and each were put into one of the three main datasets: Healthy Patients, Myocardial Infarction (MI) and Other [14]. The database was interpreted by the Hannover ECG-System (HES) to obtain the annotations for the fiducial points of each wave in each patients recording [15].

To further validate each algorithm the QT database was used [16], which contains 105 ECGs with a wide variety of QRS and ST-T morphologies, including signals from the MIT-BIH Arrhythmia Database. Out of the 105, 79 ECGs were used where the beats were manually annotated by cardiologists. Therefore, providing a database which can confirm the authenticity of the algorithms without the potential of incorrect results due to the reference annotations.

For validation of all algorithms lead V3 was chosen, due to its position being similar to the Medtronic Reveal LINQ, 
which is an implantable heart monitoring system used for cardiac diagnosis.

\section{Results}

Performance was assessed in terms of time mismatch between detections and annotations. A detection was considered correct if it fell in a window of $50 \mathrm{~ms}$ before or after the annotation. Currently, no standard position for the T-offset is confirmed, as some cardiologists state the position to be on the isoelectric line following the T-peak, whilst others state that it is located on the down slope of the wave. Therefore, this window was chosen to allow for the algorithm to detect the T-offset in a different location than where the fiducial point is, whilst still being defined correct.

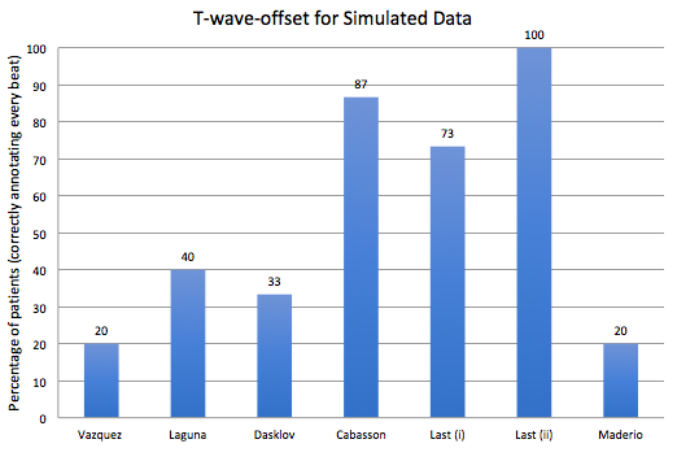

Figure 1. Bar Chart showing the percentage of recordings where the T-offset was correctly identified for each beat for the Simulated Data

The results in Figure 1 shows for every algorithm the percentage of simulated ECG recordings where the Toffset was correctly identified for every beat. Additionally, Figure 2 shows the same information but for the PTB database, with the database split into three categories: healthy, MI and other cardiac diseases.

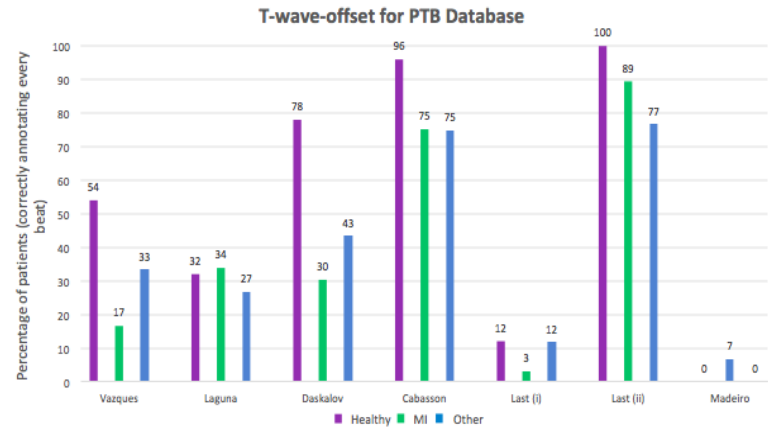

Figure 2. Bar Chart showing the percentage of recordings where the T-wave offset was correctly identified for each beat for the PTB Database
For some patients it occurred that a lot of the beats were accurately detected, however not all of them. This was specifically seen in the QT database. Therefore, in Table 1 , the mean and standard deviation of the number of beats where the T-offset was correctly identified over all patients is given for all databases.

Table 1. Mean and standard deviation values for the percentage of correctly annotated beats over all patients in each dataset

\begin{tabular}{cccccc}
\hline \hline & Simulated & Healthy & MI & Other & QT \\
\hline Vazquez & $20 \pm 41$ & $88 \pm 27$ & $46 \pm 43$ & $66 \pm 40$ & $58 \pm 25$ \\
Laguna & $64 \pm 44$ & $69 \pm 38$ & $66 \pm 41$ & $63 \pm 41$ & $34 \pm 36$ \\
Dasklov & $33 \pm 49$ & $95 \pm 16$ & $71 \pm 38$ & $72 \pm 37$ & $40 \pm 23$ \\
Cabasson & $87 \pm 35$ & $98 \pm 13$ & $92 \pm 24$ & $96 \pm 15$ & $71 \pm 24$ \\
Last (i) & $73 \pm 46$ & $32 \pm 41$ & $16 \pm 30$ & $34 \pm 40$ & $40 \pm 33$ \\
Last (ii) & $100 \pm 0$ & $100 \pm 0$ & $99 \pm 5$ & $98 \pm 7$ & $79 \pm 25$ \\
Madeiro & $33 \pm 40$ & $0 \pm 1$ & $16 \pm 35$ & $13 \pm 30$ & $28 \pm 27$ \\
\hline
\end{tabular}

\section{Discussion}

From the simulated data results it can be seen that three algorithms were able to correctly identify every beat for most of the simulated data records, with Last(ii) outperforming all with its ability to correctly identify the end for every record. It appears that four algorithms do not perform so well, typically when the T-wave is negative or has a very low amplitude. This is due to reasons such as the algorithms morphology function defining the wave with a different characteristic than it has.

Similarly, the results from the PTB database also show Cabasson and Last(ii) performing better than the other algorithms with above $75 \%$ accuracy on all patients. However, unlike with the simulated data, Last(i) is not performing well. In fact, as with the other algorithms performing badly in the simulated data, this algorithm is unable to correctly identify the T-offset if the wave is negative. Last(i) is only correctly identifying the T-peak, if it is positive, as it is found by taking the maximum of the wave. Therefore, preventing the ability to correctly annotate negative T-waves.

From this databases it can also be seen that most algorithms perform better on healthy patients, with little noise and positive T-wave morphologies. With on average $79 \pm 26$ of all T-offsets beats were correctly annotated for healthy patients, $63 \pm 28$ for MI patients and $71 \pm 21$ for patients with other cardiac diseases.

The table stating the mean and standard deviations of how many beats were correctly annotated, also confirms that two algorithms outperform the others for every database, with Last(ii) providing the best results overall. It can be seen that the algorithms are performing worse, when analysed on the Simulated and the QT database than 
the PTB. The simulated data causes issues for the algorithms due to its non smooth wave formations. The decrease in performance on the QT database can be determined as the difficulty of determining the fiducial points on patients signals, where the morphologies and characteristics of the waves are irregular. This can be seen by Figure 3 which shows 5 beats from one patient and the trouble occurring in three algorithms due to the irregularity of the recording.

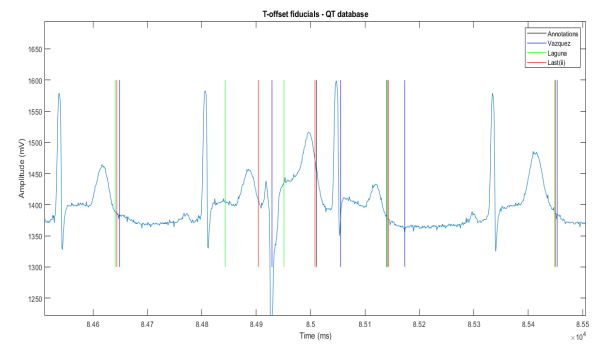

Figure 3. ECG extract from QT Database showing the T-offset detection points from three algorithms: Vazquez, Laguna and Last(ii)

This study presents the following limitations: the algorithms were not necessarily implemented as was intended by the author, causing the algorithm to not perform as expected. Furthermore, a slack window was chosen which may present a simplified look on the performance of the algorithms. However, this was seen as necessary when working on patient recordings with a range of heart rates.

\section{Conclusion}

From this study it can be said that all algorithms perform worse when annotating the T-wave for patients with cardiac diseases. This is expected due to the problems occurring with the algorithms, when the T-wave has irregular morphologies. From the results it can be seen that the algorithms which contain a method to determine the morphology of the wave or create a template which forms the shape of the wave, work better for the different morphologies occurring due to the various diseases. Whereas the other algorithms typically are only beneficial for positive T-waves. Two algorithms, Last (ii) and Cabasson, were able to have a high performance on all datasets, with above $70 \%$ accuracy, on all patients. These algorithms create templates that matches the morphology of the wave and calculate the fiducial point with the correct morphology found, causing better performance. However, with the algorithms still not having a high accuracy for all patient signals with diseases, it may not be beneficial to use them in medical applications at the moment. Therefore, it is important to continue the research and determine how to improve the algorithms to achieve a higher accuracy.

\section{References}

[1] World Health Organization, Cardiovascular Diseases, http://www.who.int/mediacentre/factsheets/fs317/en/.

[2] Zhang Q et al., An Algorithm for Robust and Efficient Location of T-Wave Ends in Electrocardiograms, IEEE transactions on bio-medical engineering, vol. 53, no. $12 \mathrm{Pt} 1$, pp. 2544-2552, 2006.

[3] Rizas K et al., Sympathetic Activity-Associated Periodic Repolarization Dynamics Predict Mortality Following Myocardial Infarction, The Journal of clinical investigation, vol. 124, no. 4, pp. 1770-1780, 2014.

[4] Manriquez A et al., Multi-lead T Wave End Detection Based on Statistical Hypothesis Testing, IFAC Proceedings Volumes, vol. 39, no. 18, pp. 93-98, 2006.

[5] Beraza I et al., Comparative Study of Algorithms for ECG Segmentation, Biomedical Signal Processing and Control, vol. 34,2017

[6] Laguna P et al., New Algorithm for QT Interval Analysis in 24-hour Holter ECG: Performance and Applications, Med. Biol Eng. Comput. 28, 1990

[7] Laguna P et al., Automatic Detection of Wave Boundaries In Multilead ECG Signals: Validation With the CSE Database, Comput. Biomed.Res. Int. J. 27, 1994

[8] Daskalov I et al., Automatic Detection of the Electrocardiogram T-Wave End. Medical \& Biological Engineering \& Computing, vol. 37, no. 3, 1999

[9] Last T et al., Multi-Component Based Cross Correlation Beat Detection in Electrocardiogram Analysis, Biomed. Eng. Online, 2004

[10] Vazquez-Seisdedos C et al., New Approach for T-Wave End Detection on Electrocardiogram: Performance in Noisy Conditions, Biomed. Eng. Online 10, 2011

[11] Madeiro J et al. New Approach for T-Wave Peak Detection and T-Wave End Location in 12-Lead Paced ECG Signals Based on a Mathematical Model. Medical Engineering \& Physics, vol. 35, no. 8, 2013

[12] Cabasson A et al., Time Delay Estimation: A New Insight Into the Woody's Method. IEEE Signal Processing Letters, vol. 15, 2008

[13] ECG Simulation Using MATLAB - MathWorks, https://nl.mathworks.com/matlabcentral/fileexchange/10858ecgsimulation-using-matlab?requestedDomain=true.

[14] Bousseljot R, et al., The PTB Diagnostic ECG Database , (1995), https://physionet.org/physiobank/database/ptbdb/

[15] HES-Corscience, https://www.corscience.com/hes

[16] Goldberger A et al., PhysioBank, PhysioToolkit, and PhysioNet: Components of a New Research Resource for Complex Physiologic Signals. Circulation 101(23):e215-e220, 2000 (June 13).

Address for correspondence:

Deborah Nairn

Kaiserstr. 12, 76131, Karlsruhe, Germany

deborah.nairn@yahoo.com 